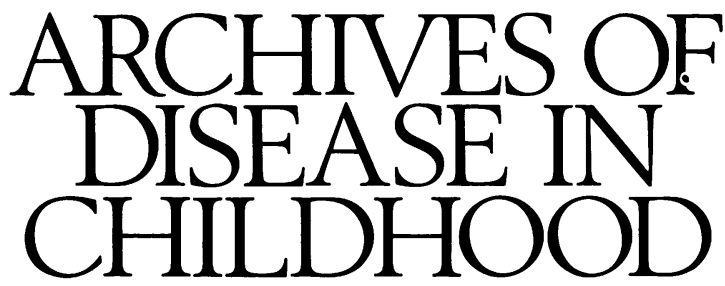

The fournal of the British Paediatric Association

\title{
Editorial
}

\section{Progress of a paper}

The journal receives about 1000 original manuscripts a year and publishes about $30 \%$. In addition we commission annotations, personal practice and current topic papers as well as a wide variety of other types of article. We publish the majority of the commissioned articles after assessment by an editor and an external reviewer or by the two editors.

\section{Original articles}

The original articles are distributed to one of the two editors or three associate editors. About $5 \%$ are rejected at this stage but the great majority are sent for peer review usually to one outside reviewer. The reviewers are selected from an extensive list or are known to be experts in the subject by the editors. We are now inviting authors, if they wish, to suggest the names of reviewers who may be competent at assessing papers in their field of interest. The two editors meet once every two weeks and each paper is accepted or rejected on the basis of the opinion of the appropriate editor or associate editor, which has been formed as a result of personal reading of the paper and the comments of the expert reviewer.

The final decision on publication is always made by the two editors who have to take into account the overall balance and direction of the journal. All papers that are considered suitable for publication are assessed at the same meeting by our statistical adviser. The reviewer or statistical adviser may raise problems that could be resolved by the author. In this case the paper is sent back to the author for further clarification. The author may satisfy the editors at a subsequent meeting and the paper is accepted. Most papers are accepted with a condition that they are revised to take account of comments by the reviewer, editor, or statistical adviser and depending on the extent of the changes required the paper may be returned to the reviewer for reassessment before a final decision is made by the editors.

The reviewers act as confidential advisers. They are asked to comment on the originality, scientific reliability, and suitability for publication in the journal. We ask them to make constructive comments to improve the paper. A good reviewer will provide suggestions that may raise the paper from an inevitable rejection to acceptance with extensive revision.

Reviewers have their names erased from reports. We consider that reviewers should not use the privilege of anonymity to be hostile or disparaging and if we recognise that this has occurred the paper is sent to another reviewer. To ensure a thorough and unbiased report we avoid sending papers to a potentially hostile camp.

We usually give the author a decision on a submitted paper within about six weeks but occasionally it takes longer. Rejected manuscripts are shredded but the comments of the reviewer and additional comments by the editor are sent to the author. Some papers are rejected but the authors are encouraged to resubmit a new version taking into account the comments of the reviewer. Occasionally an author considers that his paper was not understood by the reviewer and provides evidence that the paper was rejected unfairly. In these circumstances we are willing to have the paper assessed again.

\section{Technical editing}

The technical editor or subeditor receives the accepted final draft of the paper and prepares it for the printer. A large proportion of our readers do not have English as their first language and the technical editor has to make any changes needed to ensure that the meaning is clear. During these alterations the intentions of the author may be distorted and we rely on authors to check the proofs and to point out where this has occurred. Clarity is improved by the sparing use of semicolons, colons, brackets, hyphens, and 'dashes'. The avoidance of these punctuation marks will reduce the number of changes made by the technical editor and the possibility for error. Our statistician has asked us to avoid the \pm sign and 'NS'. Standard errors and standard deviations should be indicated. Authors should provide the $p$ value or preferably the confidence interval even if the probability does not reach the accepted level of significance. The abstract is the show piece of the paper and should be structured in content if not in style. ${ }^{1}$ Instructions to authors are published in the January and June editions of the journal and are revised regularly. Attention to these points by the author before the paper is submitted will reduce the opportunity for the introduction of errors and the time to publication.

\section{Letters and book reviews}

Letters submitted to the correspondence column are reviewed by the editors. Those raising questions or criticisms about papers published in previous editions are normally sent to the authors who are invited to reply within two to three weeks. Most published letters are not peer reviewed 
but occasionally we seek an expert opinion on those letters which are in the form of a brief scientific report. We do receive letters which take a stance on social or political issues. The decision to publish these letters rests with us as editors but we will often invite someone with alternative views to write a commentary or letter which may be published simultaneously or in a subsequent issue. About half of the letters submitted to the correspondence column are rejected by the editors. These include letters commenting on an article published more than five or six months before, letters containing trivial or unduly harsh or emotive comments on a previously published article, and those that merely report another patient with a previously published finding. Letters which do not conform to our instructions to authors stand less chance of being published because it is time consuming to request each time that a letter should be shortened or the number of references be reduced.

Book reviews are commissioned by the editors and are reviewed by them. They are not sent to outside referees as they represent the personal opinion of the author and are signed.

1 Anonymous. Writing economically. Arch Dis Child 1990;65:251.
In 1944 the Viennese paediatrician, Hans Asperger, described a group of children with what he called 'autistic psychopathy of childhood'. ${ }^{1} \mathrm{He}$ was in no doubt that this was a long lasting personality disorder, affecting predominantly boys, and he outlined its features as follows:

- Solitariness

- Abnormalities of gaze, expression, and gesture impeding emotional contact with other people

- Insensitivity to social cues

- Lack of feeling for others sometimes amounting to callousness

- Oversensitivity and insensitivity

- 'Autistic intelligence': inventive rather than imitative, with specific interests in restricted fields such as chemistry, poisons, mathematics, or art which could lead to creative achievements

- Educational delays of all kinds

- Rage or tears in the face of pressure to conform.

With age, social adaptation often improved and the work adjustment of gifted autistic psychopaths was good, but their basic personality features endured and intimate relationships remained impaired. Sexual interests were often meagre and occasionally deviant.

While Asperger considered the condition as possibly preschizophrenic, apparently only two of the 400 such cases he saw subsequently developed this illness. ${ }^{2}$ In every case one or more biological relatives were affected with the full or partial syndrome. In later years, Asperger drew a firm distinction between his 'autistic psychopathy of childhood' and Kanner's early infantile autism and he described the children, who differed from any he had seen before, as 'highly intelligent children with interesting peculiarities, yet never the less with behaviour so difficult that they were almost impossible to keep in family or school . . . They achieve the highest university professorships or become artists-yet their quirks and peculiarities will remain with them for life'. ${ }^{3}$

Two developments in diagnostic practice have occurred since then. Lorna Wing ${ }^{4}$ and Digby Tantam ${ }^{5}$ studied groups of seriously impaired children and adults (of whom only two out of 60 had married and only one had been in continuous employment since leaving school) with the features Asperger had described and found that many of their patients had had the symptoms of early infantile autism or of autism beginning in later childhood. Wing described the salient features as a failure in two way social interaction, dif- ficulty in verbal and non-verbal communication, and impaired imagination, and she coined the diagnostic label 'Asperger's syndrome'. This she held to be equivalent to high level autism (that is with normal intelligence and no gross early language delays). The concept of an 'autistic spectrum' derives from this work. ${ }^{67}$ Wing's criteria were also used by Gillberg to clarify the similarities between Asperger's syndrome and infantile autism. ${ }^{8}$

The second development arose from the identification of a group of children referred to a psychiatric clinic who described themselves or were described by others as 'loners'. They had the features of schizoid personality disorder as recorded in the older psychiatric literature (perhaps not altogether familiar to Asperger, the paediatrician). They too, while much less impaired than the cases reported by Wing and Tantam, were like the children Asperger had described. Their personality characteristics were very long lasting, ${ }^{9}$ and more recent studies showed three quarters of them to fulfil the American diagnostic criteria (Diagnostic and Statistical Manual of Mental Disorders, DSM-III) for schizotypal personality disorder which is part of the schizophrenia spectrum (S Wolff et al, to be published). The main, and enduring, features of these subjects were solitariness, impaired empathy and emotional detachment, increased sensitivity, at times with paranoid ideation, circumscribed interest patterns, and unusual styles of communication. In childhood, a very few resembled but were in fact never diagnosed as autistic children. A very few were electively mute, but most of them presented with the features of schizoid personality itself, with educational difficulties or with the kind of conduct or mixed conduct and emotional disorders usually regarded as secondary to adverse life experiences. Specific developmental delays, including delays of language related skills, were much commoner than in other referred children (S Wolff, to be published). The schizoids were predominantly boys (ratio 4:1), were of slightly above average IQ; and came from an upwardly skewed social class background. Referral took place during the school years and the main difficulty was often an apparently inexplicable failure to conform to the ordinary demands of school life. Aggressive outbursts and pathological lying were occasional features. With few exceptions these children were regarded as 'awkward' rather than handicapped.

Special school arrangements allowing for greater privacy and reduced pressure to conform were helpful, as was the 